\title{
Coloración atípica del pelaje en algunos pequeños mamíferos no voladores de Colombia
}

\author{
David Marín ${ }^{1 * \text { (iD }}$ \\ 1 Grupo de Mastozoología y Colección Teriológica Universidad de Antioquia, Calle 67 № 53 - 108, Medellín, Colombia. \\ * Correspondencia: dmarinc.academia@gmail.com
}

\section{Resumen}

Las anomalías pigmentarias en el pelaje de los mamíferos pueden estar asociadas a factores como daño folicular, baja variabilidad genética, polución, alteraciones ambientales o deficiencias dietarias. Documentar este patrón es importante pues el pelaje es uno de los caracteres más importantes del sistema tegumentario en este grupo de animales. En Colombia el piebaldismo, ha sido reportado sólo en una especie de pequeños mamíferos no voladores. Es así como en esta nota se registra el fenómeno en seis especies pertenecientes a los órdenes Rodentia, Eulipotyphla y Paucituberculata.

Palabras clave: Piebaldismo, Paucituberculata, Rodentia, Eulipotyphla

\begin{abstract}
Pigmentary abnormalities in mammalian fur can be associated with factors such as follicular damage, low genetic variability, pollution, environmental changes, or dietary deficiencies. Documenting this pattern is important, since fur is one of the most important characters of the integumentary system in this group of animals. In Colombia, piebaldism has been registered in just one small mammal species. Thus, in this note the phenomenon is recorded in six species belonging to orders Rodentia, Eulipotyphla and Paucituberculata.
\end{abstract}

Key words: Piebaldism, Paucituberculata, Rodentia, Eulipotyphla

Uno de los caracteres más importantes y distinguibles del sistema tegumentario de los mamíferos es el pelo, el cual presenta diferentes formas (e.g. púas, cerdas, escamas, placas, etc.) y funciones (e.g. termorregulación y defensa). Anexo a la estructura, el pelo resalta una importante característica funcional: la coloración. Esta cualidad es muy variable en la clase Mammalia y puede informar acerca de la edad, el estado reproductivo, el sexo, estacionalidad, enfermedades, e incluso ayuda a diferenciar poblaciones y es una gran herramienta en la taxonomía (Stoner et al. 2003; Caro 2005).

Conocer los cambios anormales en el pelaje de los mamíferos es importante y debe ser documentado. Diferentes variantes en la coloración (albinismo parcial o total, leucismo, xantismo, piebaldismo, etc.), han sido detalladamente ilustradas en roedores (e.g. Bowman \& Curran 2000), musarañas (e.g. Gelling 2003), murciélagos (e.g. Ruelas et al. 2016), 


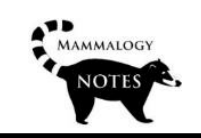

carnívoros (Acevedo \& Aguayo 2008), xenartros (Lopes et al. 2019), entre otros. Para la mayoría de estos trabajos se ha utilizado de manera imprecisa los términos albinismo parcial que se presenta como parches de color blanco asociado a la ausencia de melanina en la piel y ojos (Barquez et al. 2003) y leucismo, que se refiere a la condición en donde hay una reducción de la pigmentación parcial o total que mantiene la coloración normal de los ojos y/o la piel (Rivera-Prieto \& Marín-C 2017). Sin embargo, utilizar estos términos para referirse al patrón de manchas o parches blancos en diferentes zonas del cuerpo es considerado obsoleto y el correcto término es piebaldismo (Lamoreux et al. 2010; Lucati \& López-Baucells 2017; Veloso-Frías et al. 2020).

A pesar de ser reportados en una amplia variedad de grupos de vertebrados, los fenómenos de piebaldismo son raros (Boada \& Tirira 2010). En Colombia esta anomalía ha sido registradas en los órdenes Rodentia (Montoya-Bustamante et al. 2017), Chiroptera (Roncancio \& Ramírez-Chaves 2008; Marin-Vasquez et al. 2010; Mantilla-Meluk \& JiménezOrtega 2011; Marín-Vásquez et al. 2013; Velandia-Perilla 2013; Olarte-Gonzalez et al. 2014; Chacón et al. 2015) y Artiodactyla (Lehman 1959). Sin embargo, los registros en otros órdenes de mamíferos pequeños no voladores (PMNV) no están disponibles. Por tal razón, el objetivo de esta nota es dar a conocer el fenómeno en algunas especies de los órdenes Rodentia, Eulipothypla y Paucituberculata en el país.

El análisis se desarrolló mediante la inspección directa de especímenes depositados en la colección de mastozoología del Instituto de Ciencias Naturales Alberto Cadena (ICN) y la Colección Teriológica Universidad de Antioquia (CTUA). Ocho registros de piebaldismo fueron encontrados en tres órdenes de PMNV, en las especies Akodon affinis, Necromys urichi, Handleyomys fuscatus, Cryptotis colombianus, Cryptotis thomasi y Caenolestes fuliginosus (Figura 1). Los especimenes no presentaron ningún otro tipo de anomalía morfológica, y redimieron lo enunciado en la diagnosis craneodental (Gardner 2007; Patton et al. 2015).

En Suramérica, anomalías de coloración se han registrado en 16 especies de PMNV en Brasil, Chile, Colombia, Ecuador y Perú que sumados a este aporte, genera un total de 66 individuos agrupados en 21 especies (Pessoa \& Dos-Reis 1995; Cademartori \& Pacheco 1999; Ramirez \& Arana 2005; Neves et al. 2014; Brito \& Valdivieso-Bermeo 2016; MontoyaBustamante et al. 2017; Veloso-Frías et al. 2020). En musarañas (Soricidae) y ratones runchos (Caenolestidae), es la primera vez que el piebaldismo se registra en el continente. La ocurrencia de este fenómeno está asociada probablemente a factores como daño tisular (Phillips 1954), baja variabilidad genética (Jehl Jr 1985), alteraciones ambientales (Moller \& Mousseau 2001) o deficiencias dietarias (Clapp 1974) (este último cuando hay una marcada simetría e.g. C. fuliginosus).

A. affinis con tres individuos registrados en la misma localidad genera cuestionamientos acerca de la incidencia y las causas del fenómeno. Al parecer, el piebaldismo podría ser más común en poblaciones pequeñas y aisladas (Chętnicki et al. 2007) ya que la endogamia aumenta la probabilidad de que alelos recesivos se expresen (Bensch et al. 2000). El porcentaje de aparición de la anomalía para ratones de campo, oscila entre el 1,8\% y 15,4\%, mientras que en las musarañas del género Blarina, el porcentaje oscila entre un 0,50\% y 5\% en Norteamérica (Moncrief \& Anderson 1997; Francl \& Warnell 2003). Para la especie A. affinis, Montoya-Bustamante et al (2017) registran individuos con manchas blancas de menor extensión en comparacion a los reportados en esta nota, en localidades ubicadas en el Parque Natural Regional Ucumarí, cercanas al Santuario Otún Quimbaya. Ellos sugieren una mayor prevalencia del fenómeno en la cordillera central de los Andes, en 
donde los machos de estos roedores presentan mayor incidencia. Los datos registrados en esta nota soportan la necesidad de incluir a la especie A. affinis dentro de estudios de genética poblacional para establecer si presenta endogamia, especialmente en localidades donde el piebaldismo ha sido reportado.
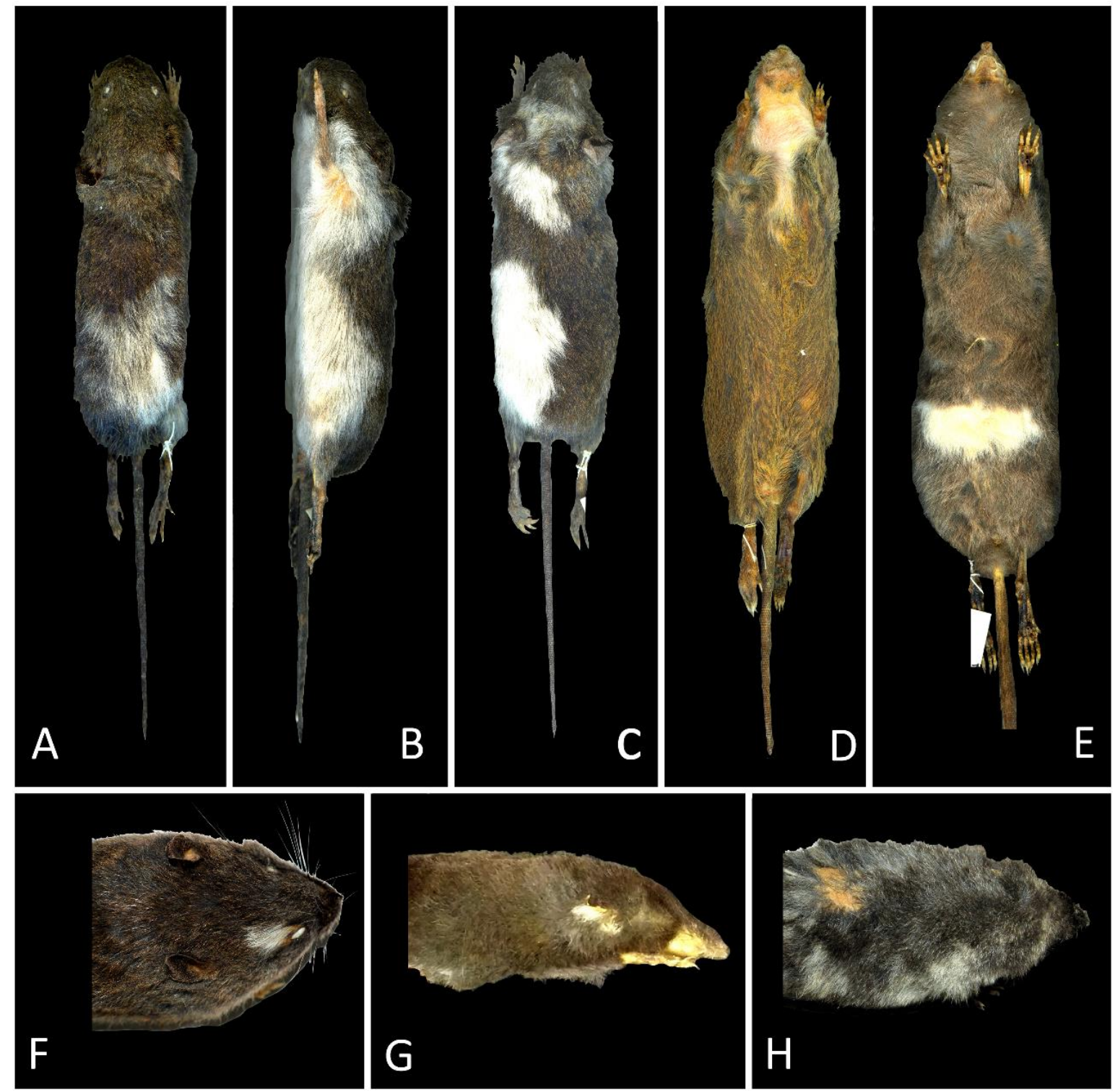

FIGURA 1. Coloración atípica en el pelaje de mamíferos pequeños no voladores de Colombia. A, B, C Akodon affinis, Risaralda, Pereira, Santuario de Fauna y Flora Otún Quimbaya (ICN 126010 (vista dorsal), ICN 126049 (vista lateral), ICN 12606 (vista dorsal), D. Necromys urichi Meta, Fuentedeoro (ICN 91050') (vista ventral), E. Caenolestes fuliginosus, Cundinamarca, Junín, Reserva Biológica Carpanta (ICN 111130') (vista ventral), F. Handleyomys fuscatus, Antioquia, municipio de Jardín, vereda la Mesenia, Fundación Colibrí (CTUA JMC 1770') (vista rostro dorsal), G. Cryptotis colombianus, Antioquia, municipio de Medellín (CTUA 3638) (vista rostro lateral), H. Cryptotis thomasi, Cundinamarca, municipio de Suesca (MUJ 1098 (Museo Universidad Javeriana)) (vista rostro dorsal); este ejemplar fue revisado en la CTUA. 
Se ha sugerido que los individuos piebaldistíscos pueden ser más susceptibles a la depredación y una sobrevivencia reducida debido a rasgos patológicos como defectos sensoriales o nerviosos, anemia, baja fertilidad, mayor susceptibilidad a enfermedades, baja visión, o un bajo reconocimiento en las interacciones con los conespecíficos (Acevedo \& Aguayo 2008). El fenómeno en esta nota se reporta en ejemplares adultos, y sumado a los casos de juveniles en PMNV (Brito \& Valdivieso 2016), podría indicar que el pielbaldismo no afecte la sobrevivencia hasta la adultez. Sin embargo, la tasa a la cual se registran estas anomalias y los estudios de genética de poblaciones relacionados no permiten una determinación precisa de la influencia de la alteración en el fitness de los individuos.

Esta nota muestra evidencia de como en las colecciones biológicas se encuentra información importante de historia natural, relacionadas directamente con el estado poblacional de algunas especies de mamíferos, las cuales pueden estar sujetas a procesos de endogamia por fragmentación de hábitat. Además, espera estimular la revisión y reporte de este y otros tipos de fenómenos en otras colecciones de referencia y sumarlos a datos genéticos que ayuden a explicar el origen y las repercusiones de las aberraciones cromáticas en los mamíferos del neotrópico.

\section{AGRADECIMIENTOS}

Yaneth Muñoz por permitir el acceso a los especímenes de la Colección de mamíferos del Instituto de Ciencias Naturales Alberto Cadena, Marcela Gómez Laverde, Hugo López, Berta Calonge, Edilson Patiño, Sergio Solari curador de la Colección Teriológica Universidad de Antioquia, Alejandra Bonilla, Román Bonilla, Ángela Viviana Rojas.

\section{REFERENCIAS}

Acevedo J, Aguayo M. 2008. Registro en Chile de leucismo en lobo marino común, con una revisión de las coloraciones anormales en otáridos. Revista de biología marina y oceanografía. 43(2):413-417. http://doi.org/10.4067/S0718-19572008000200017

Barquez RM, Carrizo L V., Ferro LI, Flores DA, Mollerach MI, Sánchez. MS, López APG. 2003. Primer caso de albinismo total para Sturnira erythromos. Chiroptera Neotropical: 166-169.

Bensch S, Hansson B, Hasselquist D, Nielsen B. 2000. Partial albinism in a semi-isolated population of great reed warblers. Hereditas. 133(2):167-170.

Boada C, Tirira DG. 2010. First record of partial albinism (leucism) in Carollia perspicillata (Phyllostomidae) in Ecuador. Chiroptera Neotropical. 16:755-757.

Bowman J, Curran RM. 2000. Partial Albinism in a Red-Backed Vole, Clethrionomys gapperi, from New Brunswick. Northeastern Naturalist. 7(2):181. https:// doi.org/10.2307/3858650

Brito J, Valdivieso-Bermeo K. 2016. First records of leucism in eight species of small mammals (Mammalia: Rodentia). Therya. 7(3):483-489. https:// doi.org/10.12933/therya-16-408

Cademartori C V, Pacheco SM. 1999. Registro de albinismo em Delomys dorsalis (Hensel, 1872) (Cricetidae, Sigmodontinae). Biociências. 7(1):195-197.

Caro T. 2005. The Adaptive Significance of coloration in animals. Bioscience. 55(2). https:// doi.org/10.1641/0006-3568(2005)055[0125:TASOCI]2.0.CO;2 
Chacón PJ, Charrasquiel, González Carlos M, Correa BJ. 2015. Registro de leucismo en Artibeus planirostris (Chiroptera: Phyllostomidae) en Colombia. Acta Zoologica Mexicana, n.s,. 31(1):125-128.

Chętnicki W, Fedyk S, Bajkowska U. 2007. Cases of coat colour anomalies in the common shrew, Sorex $\begin{array}{llll}\text { araneus L. Folia } & \text { Biologica } & \text { (Praha). }\end{array}$ https:// doi.org/10.3409/173491607780006308

Francl KE, Warnell DB. 2003. Pelage anomaly in a northern short-tailed shrew, Blarina brevicauda, from West Virginia. Banisteria. 22:59-60.

Gardner AL. 2007. Mammals of South America. The University of Chicago Press.

Gelling M. 2003. Partial albinism in the common shrew Sorex araneus. Mammal Review. 33(2):189190. https:// doi.org/10.1046/j.1365-2907.2003.00014.x

Lamoreux M, Delmas V, Laure L, Bennett DC. 2010. The color of mice. A model genetic network. 1st Edition. Lamoreux M, Delmas V, Laure L, Bennett DC, editors. Wiley-Blackwell, Texas.

Lehman FC. 1959. Pudu mephistophiles wethmorei. Novedades Colombianas. 1(4):202-204.

Lopes AC, Junior E, de Freitas MA, Molina K. 2019. Primeiro registro de leucismo em Bradypus variegatus (Schinz 1825, Xenarthra: Bradypodidae), município de Maceió, Alagoas, Brasil. Edentata (20):39-43. https://doi.org/10.2305/iucn.ch.2019.edentata-20-1.8.en

Lucati F, López-Baucells A. 2017. Chromatic disorders in bats: a review of pigmentation anomalies and the misuse of terms to describe them. Mammalian Review. 47(2):112-123. https://doi.org/10.1111/mam.12083

Mantilla-meluk H, Jiménez-ortega AM. 2011. First case of albinism in Uroderma bilobatum and its implications in the evolution of coat color patterns among Vampyressine bats. Investigacion Biodiversidad y Desarrollo. 30(2):97-100.

Marín-Vásquez A, González-Ibarra I, Gualy L, Díaz J. 2013. Aberrations in coat color pattern of colombian bats: isabelline, leucism and melanocytes nevus. Online Journal of Biosciences and Informatics. 4:253-265.

Marin-Vasquez A, Ortega-Rincón M, ramírez-Chaves HE. 2010. Records of leucism in three species of Colombian bats: Carollia brevicauda, Artibeus jamaicensis and Lophostoma silvicolum (Phyllostomidae). Chiroptera Neotropical. 16(2):706-709.

Moncrief ND, Anderson JM. 1997. White-belted coloration in a northern short-tailed shrew (Blarina brevicauda). American Midland Naturalist. 137(2):397-400. https:// doi.org/10.2307/2426859

Montoya-Bustamante S, Zapata-Mesa N, Murillo-García OE. 2017. Leucism in Akodon affinis (Allen, 1912) (Rodentia: Cricetidae). Therya. 8(3):269-272. https://doi.org/10.12933/therya-17-494

Neves AC da SA, Coutinho LC, Oliveira MB de, Leila Maria Pessôa. 2014. First report of partial albinism in genus Thrichomys (Rodentia: Echimyidae). Papéis Avulsos de Zoología. 54(9):107-110. http://www.scielo.br/pdf/paz/v54n9/a01v54n9.pdf

Olarte-Gonzalez G, Vela-Vargas IM, Camargo P, Jhonson T, Jiménez Á. 2014. Primer Reporte de leucismo en Artibeus lituratus (Olfers, 1818) (Phyllostomidae: Stenodermatinae) en el Caribe Colombiano. Mammalogy Notes. 1(1):7-8. https:/ / doi.org/10.47603/manovol1n1.7-8

Patton JL, Pardiñas UF, D’Elía G. 2015. Mammals of South America. Volume 2: University of Chicago Press. 
Pessoa LM, Dos-Reis SF. 1995. Coat color variation in Proechimys albispinus (Geoffroy, 1838) (Rodentia, Echimyidae). Boletim do Museu Nacional, Nova Série Zoologia. 361:1-5.

Ramirez OE, Arana M. 2005. Albinism in the andean leaf-eared mouse, Phyllotis andinum (Rodentia, Cricetidae). Mastozoología Neotropical. 12(2):269-270.

Rivera-Prieto D, Marin-C D. 2017. Atypical colororation in the yellow-striped poisonous frog, Dendrobates truncatus (Cope, 1861), in the Colombian Magdalena river valley. Cuadernos de Herpetología. 31(1):33-35. https:// doi.org/10.31017/9607

Roncancio N, Ramírez-chaves HE. 2008. Registro de leucismo en Sturnira erythromos en los Andes centrales de Colombia. Chiroptera Neotropical. 14(2).

Ruelas D, Ramos M, Molina M, Pacheco V. 2016. First record of leucism in Glossophaga soricina valens (Phyllostomidae: Chiroptera) from Peru. Therya. 7(2):343-352. https:// doi.org/10.12933/therya-16-381

Stoner CJ, Bininda-Emonds ORP, Caro T. 2003. The adaptive significance of coloration in lagomorphs. Biological Journal Linnean Society. 79(2):309-328. https:// doi.org/10.12933/therya-16-381

Velandia-Perilla JH. 2013. Leucismo En Murciélagos De Hoja Nasal (Chiroptera, Phyllostomidae) De Colombia. Boletín Científico. Centro de Museos. Museo de Historia Natural. 17(2):87-94.

Veloso-Frías J, Moreno L, Silva-De la Fuente M, Najle M, Torres-Fuentes L, Doussang D, Ardiles K, González-Acuña D. 2020. Piebaldismo en dos especies de roedores sigmodontinos del Parque Nacional Torres del Paine, Chile. Notas sobre Mamíferos Sudamericanos. 01(1):001007. http:// doi.org/10.31687/saremNMS.20.0.24

Editor: Héctor Ramírez-Chaves

Recibido 2020-08-08

Revisado: 2020-08-25

Aceptado 2020-12-04

Publicado 2021-02-02 